\title{
Early Stage Triple Negative Breast Cancer Has Significantly Better Outcomes than More Advanced Disease: A Single Centre Retrospective Review
}

\author{
Caroline Hamm ${ }^{1 *}$, Swati Kulkarni ${ }^{1}$, Rasna Gupta ${ }^{1}$, Amin Kay ${ }^{1}$, John Mathews ${ }^{1}$, Khalid Hirmiz ${ }^{1}$, \\ Indryas Woldie', Akmal Ghafoor ${ }^{1}$, Tarek Elfiki', Sindu Kanjeekal1, Ming Pan1, Kenneth Schneider ${ }^{1}$, \\ Junaid Yousuf ${ }^{1}$, Mohammad Jarrar ${ }^{1}$, Colvin Springer ${ }^{1}$, Maher El-Masri ${ }^{2}$
}

${ }^{1}$ Schulich School of Medicine, Western University, London, ON, Canada

${ }^{2}$ University of Windsor, Windsor, ON, Canada

Email: *caroline.hamm@wrh.on.ca

How to cite this paper: Hamm, C., Kulkarni, S., Gupta, R., Kay, A., Mathews, J., Hirmiz, K., Woldie, I., Ghafoor, A., Elfiki, T., Kanjeekal, S., Pan, M., Schneider, K., Yousuf, J., Jarrar, M., Springer, C. and ElMasri, M. (2016) Early Stage Triple Negative Breast Cancer Has Significantly Better Outcomes than More Advanced Disease: A Single Centre Retrospective Review. Journal of Cancer Therapy, 7, 665-674.

http://dx.doi.org/10.4236/jct.2016.710069

Received: April 27, 2016

Accepted: September 19, 2016

Published: September 22, 2016

Copyright $\odot 2016$ by authors and Scientific Research Publishing Inc. This work is licensed under the Creative Commons Attribution International License (CC BY 4.0).

http://creativecommons.org/licenses/by/4.0/

\section{(c) (i) Open Access}

\begin{abstract}
A retrospective, serial analysis of 181 triple negative breast cancer (TNBC) patients was undertaken at a regional cancer centre in Canada. The primary focus of the analysis was to investigate the effect of presenting stage in patients with TNBC on progression free and overall survival. We were able to demonstrate that patients presenting with an earlier stage breast cancer had a significantly superior progression free and overall survival when compared to more advanced stage. The adjusted multivariate cox-regression analyses for the overall and progression free survival suggest that the hazard of death was significantly lower for patients with stages I (HR $=0.09$; $95 \%$ CI $0.03-0.24)$ and II ( $\mathrm{HR}=0.29 ; 95 \%$ CI 0.16 - 0.54) than for patients with stage III. The only other predictor of progression free survival besides stage, was receipt of radiotherapy ( $\mathrm{HR}=0.39 ; 95 \% \mathrm{CI} 0.22-0.69)$ in the adjusted cox regression analysis. Less than $2 \%$ of patients presented with stage IV disease. The small numbers presenting with stage IV disease may have impact on the development of clinical and translational trials. Certainly there may be stage migration if staging included more standardized or more sensitive investigations such as PET scans, and this might an important consideration in developing clinical trials. Twenty-five percent of patients presented with stage I disease. It is important for patients with TNBC presenting with earlier stages of disease that they are aware that they will have a better prognosis than their counterparts with more advanced disease. It is important that we are aware of this patient population, as their treatment recommendations are unclear and a source of a fair amount of controversy currently.
\end{abstract}




\section{Keywords}

Triple Negative Breast Cancer, Prognostic Factors, Stage

\section{Introduction}

Triple negative breast cancer (TNBC) remains clinically defined as estrogen receptor (ER) negative, progesterone receptor (PR) negative and human epidermal growth factor 2 (Her-2) negative. The vast majority of TNBC are high-grade invasive ductal carcinomas of no special type. TNBC carries significant heterogeneity by pathology as measured by molecular signature. Molecular subtypes have been divided into basal-like; immunomodulatory, mesenchymal-like [1] [2]. In addition, TNBC is often associated with high levels of Ki67 (a proliferation marker) [2]. Tumor Infiltrating Lymphocytes are also being investigated as an important predictive and or prognostic factor in TNBC [3].

Overall, patients with TNBC are considered to have an inferior outcome than those patients that have the option of targeted therapies [4]-[7]. However, few previous studies have differentiated outcomes in this patient population by presenting stage, which is known to be one of the most important predictors of outcome in breast cancer. Stage is a reported prognostic factor in the TNBC patient group [8]-[10]. Further, very little is known about whether or not chemotherapy is warranted at all stages of TNBC. Thus, the primary purposes of this study were to 1) compare the overall and progression free survival of TNBC across the various stages of cancer and 2) to compare the overall and progression free survival of these patients based on their chemotherapy status across each cancer stage. A secondary purpose of our study was to explore the frequency of TNBC recurrence among patients with stage 1 cancer across chemotherapy and surgery.

\section{Methods}

\subsection{Data Collection}

Upon clearance by the Institutional Research Ethics Board, we conducted a retrospective chart review of all patients who were diagnosed with TNBC at our regional cancer center between 2004 and 2010. This review yielded a sample of 181 eligible patients. We elected to limit our starting date to 2004 because testing for Her 2 at our center was first introduced as a standard test in 2003. Patients were treated according to current standard of care. Treatment options included dose dense adriamycin, cyclophosphamide and taxol (dose dense ACT), 5FU, epirubicin, cyclophosphamide, taxotere (FEC-D) primarily, per standard of care. Some elderly patients did not receive chemotherapy because of comorbidities.

\subsection{Pathology Assessment}

The ER and PR analyses used Novocastra antibodies (Leica Microsystems, Concord, 
ON), with cut-off levels for receptor positivity of more than or equal to $10 \%$. Although ER and PR positivity are considered at more than $1 \%$ in current clinical practice, $10 \%$ was the accepted level during the reported time period. In many charts, results were reported only as positive or negative; details of percentage positivity were omitted. Positivity for overexpression of her2 was evaluated using the Novocastra CB11 monoclonal antibody (Leica Microsystems). Results were reported as 1+ to 3+ density of staining. A result of $3+$ was reported as positive, 2+ was reported as indeterminate, and 1+ was reported as negative. Tumours reported as $2+$ were then tested for gene overexpression using fluorescence in situ hybridization (fish). Threshold for a positive her2 fish result was a her2-to-CEP17 ratio exceeding 2.2. We started our analysis in 2014, as this is when her2 testing was done routinely at our centre.

\subsection{Data Analysis}

Data were analyzed using the IBM.SPSS statistical package version 22. Prior to data analysis, all data points were explored for accuracy and violations of statistical assumptions. Descriptive statistics were performed to describe the demographic and prognostic characteristics of the sample, and to compare these characteristics by stage of cancer. Kaplan Meier analysis was performed to compare the overall and progression free survival across cancer stages and to compare those who received chemotherapy and those who did not across each stage. Multivariate cox regression analysis was performed to explore the adjusted association between cancer stage and survival (overall and progression free survival). To preserve the parsimony of the regression model and avoid the potential of multicollinearity, the stage and cancer grade. All analyses were performed using a two tailed alpha of 0.05 and statistical significance was ascertained based on a p value of $\leq 0.05$ or $95 \%$ confidence interval (95\% CI).

\section{Results}

\subsection{Sample Characteristics}

The initial sample for this study was 181 patients with a confirmed diagnosis of TNBC. Of those, only 2 patients presented with stage 4 cancer, and thus were deleted from the analysis (not enough to compare against stages 1,2, and 3), yielding a sample size of 179 patients. Forty-four (24.6\%) patients were classified as stage 1, 94 (52.5\%) were classified as stage 2 , and $41(22.9 \%)$ were classified as stage 3 patients. Table 1 displays the demographic and prognostic characteristics of our sample compared across these three stages. The data show that patients were not different in their age, family history of cancer, radiation therapy or their cancer grade. Chemotherapy however was significantly lower among patients with stage 1 cancer (59.1\%) as compared to patients with stages $2(79.8 \%)$ and $3(95.1 \% ; \mathrm{p}<0.001)$. In addition, none of the patients with stage 1 cancer received hormonal therapy compared to $6.4 \%$ from stage 2 and $7.3 \%$ from stage 3. No statistical difference was observed among the three groups with regard to their rate of hormonal therapies.

Forty-four patients were stage I. Twenty-six (59.1\%) received chemotherapy and $18 \mathrm{did}$ 
Table 1. The demographic and prognostic characteristics of our sample compared across these three stages of triple negative breast cancer.

\begin{tabular}{|c|c|c|c|c|c|c|}
\hline \multirow{2}{*}{ Variable } & \multicolumn{3}{|c|}{ Stage of Cancer } & \multirow{2}{*}{$\begin{array}{c}\text { Total } \\
(\mathrm{N}=185)\end{array}$} & \multirow{2}{*}{$\chi^{2} / F$} & \multirow{2}{*}{$\mathrm{p}$} \\
\hline & $1(n=46)$ & $2(n=96)$ & $3(n=43)$ & & & \\
\hline Age $[\mathrm{m} \pm \mathrm{SD}]$ & $58.36 \pm 13.32$ & $55.84 \pm 13.09$ & $53.95 \pm 11.95$ & $56.10 \pm 12.94$ & 1.50 & 0.23 \\
\hline Size of cancer $[\mathrm{m} \pm \mathrm{SD}]$ & $1.46 \pm 0.50$ & $2.98 \pm 1.01$ & $3.30 \pm 1.58$ & $2.68 \pm 1.29$ & 39.79 & $\leq 0.001$ \\
\hline Personal history of cancer [n (\%)] & & & & & 0.44 & 0.80 \\
\hline No & $45(97.8)$ & $93(96.9)$ & $41(95.3)$ & $179(96.8)$ & & \\
\hline Yes & $1(2.2)$ & $3(3.1)$ & $2(4.7)$ & $6(3.2)$ & & \\
\hline Family history of cancer [n (\%)] & & & & & 0.33 & 0.85 \\
\hline No & $10(21.7)$ & $22(22.9)$ & $8(18.6)$ & $40(21.6)$ & & \\
\hline Yes & $36(78.3)$ & $74(77.1)$ & $35(81.4)$ & $145(78.4)$ & & \\
\hline Grade of cancer [n (\%)] & & & & & 1.51 & 0.47 \\
\hline Grade 1 - 2 & $14(30.4)$ & $21(21.9)$ & $9(20.9)$ & $44(23.8)$ & & \\
\hline Grade 3 & $32(69.6)$ & $75(78.1)$ & $34(79.1)$ & $141(76.2)$ & & \\
\hline Hormonal therapy [n (\%)] & & & & & 0.25 & 0.88 \\
\hline No & $42(91.3)$ & $87(90.6)$ & $38(88.4)$ & $167(90.3)$ & & \\
\hline Yes & $4(8.7)$ & $9(9.4)$ & $5(11.6)$ & $18(9.7)$ & & \\
\hline Chemotherapy & & & & & 17.51 & $\leq 0.001$ \\
\hline No & $19(41.3)$ & $20(20.8)$ & $2(4.7)$ & $41(22.2)$ & & \\
\hline Yes & $27(58.7)$ & $76(79.2)$ & $41(95.3)$ & $144(77.8)$ & & \\
\hline Radiation therapy [n (\%)] & & & & & 4.06 & 0.132 \\
\hline Yes & $20(43.5)$ & $33(34.4)$ & $10(23.3)$ & $63(34.1)$ & & \\
\hline No & $26(56.5)$ & $63(65.6)$ & $33(76.7)$ & $122(65.9)$ & & \\
\hline
\end{tabular}

not (40.9\%). The median age of those that declined chemotherapy was 69 years of age.

All patients in our sample underwent some type of surgical intervention. The numbers were too small to determine influence of surgical choice in treatment. Variable "cancer size in centimetres" was not included in the cox regression analysis because it is redundant with cancer.

\subsection{Unadjusted Survival Analysis}

The Kaplan Meier results in Table 2 suggest that the overall survival and progression free survival were both significantly different across the three cancer stages, with patients in stage 1 experiencing the longest mean survival (9.66 years) as compared to 7.92 and 5.21 years for stages 2 and 3 respectively. However, median survival was only calculable for patients in stage 2 and 3.

As well, presenting stage was a significant predictor of progression free survival ( $\mathrm{p}<$ $0.001)$ and overall survival $(p<0.001)$. In addition to this, receipt of chemotherapy was a significant predictor of progression free survival for stage II patients $(\mathrm{p}=0.01)$, with a trend toward predicting overall survival in this stage of patients $(p=0.09)$. Figure $1 \&$ Figure 2 display the progression free and overall survival curves associated with these results. 
Table 2. Kaplan Meier comparisons across stages of cancer and by stage across chemotherapy status.

\begin{tabular}{|c|c|c|c|c|c|c|c|c|c|}
\hline \multirow{2}{*}{ Variable } & \multirow{2}{*}{$\mathrm{N}(\%)$} & \multicolumn{4}{|c|}{ OS in Years } & \multicolumn{4}{|c|}{ PFS in Years } \\
\hline & & Mean & Median & $\log \operatorname{Rank} \chi^{2}$ & $\mathrm{P}$ & Mean & Median & $\log \operatorname{Rank} \chi^{2}$ & $\mathrm{p}$ \\
\hline Overall & & & & 24.10 & $<0.001$ & & & 25.03 & $\leq 0.001$ \\
\hline Stage 1 & $46(24.90)$ & 7.42 & --- & & & 7.41 & --- & & \\
\hline Stage 2 & $96(51.90)$ & 6.35 & --- & & & 6.36 & --- & & \\
\hline Stage 3 & $43(23.20)$ & 4.04 & 3.00 & & & 3.75 & 3.00 & & \\
\hline Stage 1 only & & & & 0.25 & 0.62 & & & 2.68 & 0.10 \\
\hline No Chemo & $19(41.30)$ & 4.63 & & & & & -- & & \\
\hline Chemo & $27(58.70)$ & 7.53 & & & & & -- & & \\
\hline Stage 2 only & & & & 11.77 & $\leq 0.001$ & & & 0.07 & 0.79 \\
\hline No Chemo & $20(20.80)$ & 4.01 & & & & 4.84 & & & \\
\hline Chemo & $76(79.20)$ & 6.78 & & & & 6.39 & & & \\
\hline Stage 3 only & & & & 3.36 & .07 & & & 0.57 & 0.81 \\
\hline No Chemo & $2(4.70)$ & 1.00 & 1.00 & & & 0.50 & 6.00 & & \\
\hline Chemo & $41(95.30)$ & 4.19 & 5.00 & & & 50.33 & 3.77 & & \\
\hline
\end{tabular}

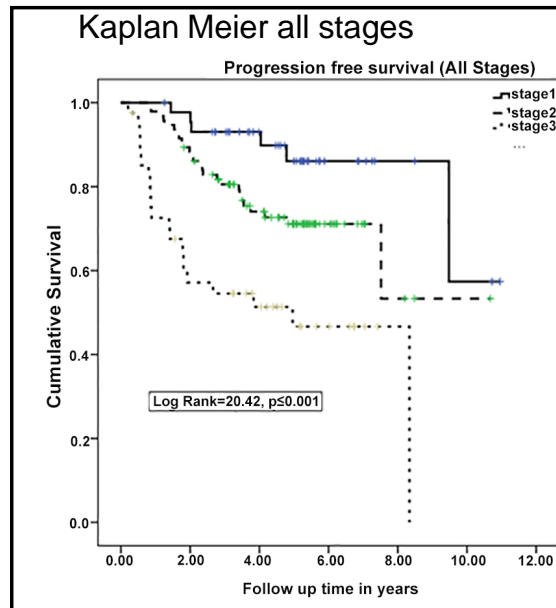

Kaplan Meier stage 2 across chemo Rx

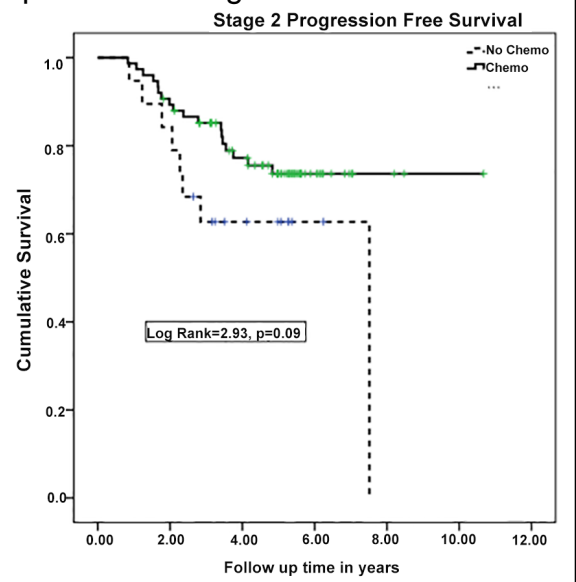

Kaplan Meier stage 1 across chemo Rx

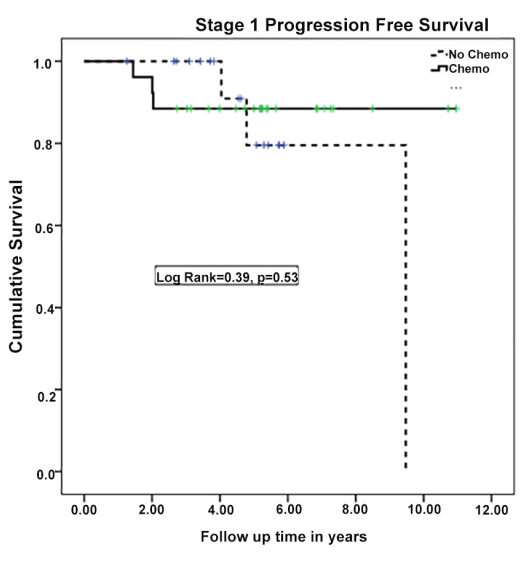

Kaplan Meier stage 3 across chemo Rx

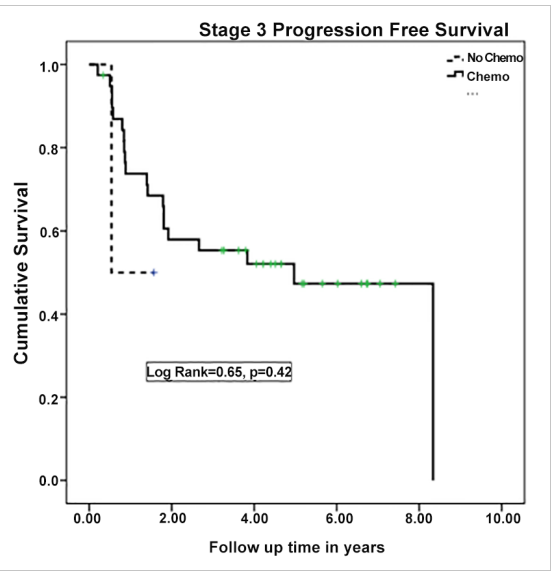

Figure 1. Progression-free survival, outcome by stage and receipt of chemotherapy. 


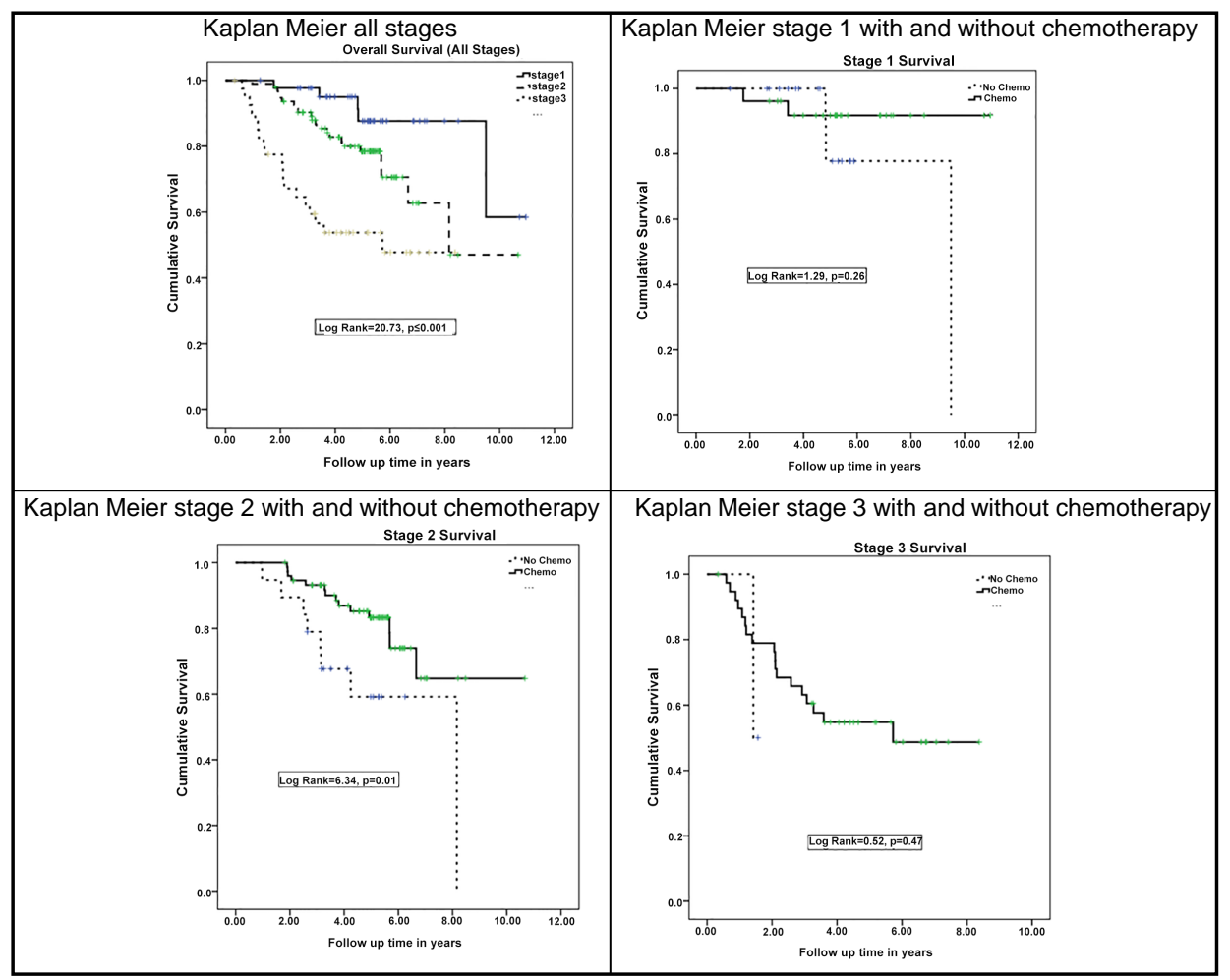

Figure 2. Overall survival, outcome by stage and receipt of chemotherapy.

\subsection{Adjusted Multivariate Analysis}

Table 3 displays the adjusted multivariate cox-regression analyses for the overall and progression free survival. The results suggest that the hazard of death was significantly lower for patients with stages I ( $\mathrm{HR}=0.09 ; 95 \% \mathrm{CI} 0.03-0.24)$ and II $(\mathrm{HR}=0.29 ; 95 \%$ CI 0.16 - 0.54) than it is for patients with stage III The only other predictor of progression free survival besides stage, was receipt of radiotherapy ( $\mathrm{HR}=0.39$; 95\% CI 0.22 0.69 ) in the adjusted cox regression analysis. None the measured variables were significant predictors of overall survival

\section{Discussion}

Median age was 56 years $(27-85)$, which is slightly older than some previously published studies [4] [11], and still younger than the median of non-TNBC breast cancer of 60.9 years [10]. Almost $40 \%$ per cent of our patient population was over the age of 60 . The age groups 40 - 59 included $53 \%$ of the TNBC population.

Overall incidence of the TNBC population in our dataset was $12.7 \%$ (181/1423 total patients), which may be smaller than in published series [4] [6] [9] [12]. The catchment area for this cancer centre carries a lower percentage of African Americans and Hispanic patients, which could account for this study's findings.

Only 2 of the 181 patients presented with stage IV disease. This is similar to the $3.9 \%$ presented in the California Cancer Registry [13]. Certainly there may be stage migration if staging included more sensitive investigations such as PET scans, and this might 
Table 3. The adjusted multivariate cox-regression analyses for the overall and progression free survival for the triple negative population

\begin{tabular}{|c|c|c|c|c|c|c|c|c|c|}
\hline \multicolumn{6}{|c|}{ Overall Survival in Months } & \multicolumn{4}{|c|}{ PFS in Months } \\
\hline Variable & $\mathrm{N}(\%)$ & Mean & $\begin{array}{c}\text { Median } \\
\text { (n) }\end{array}$ & $\log \operatorname{Rank} \chi^{2}$ & $\mathrm{p}$ & Mean & Median & $\log \operatorname{Rank} \chi^{2}$ & $\mathrm{p}$ \\
\hline Overall & & & & 24.84 & $<0.001$ & & & 22.47 & $<0.001$ \\
\hline Stage I & $46(24.9)$ & 93.77 & $\ldots .$. & & & 93.9 & $\ldots .$. & & \\
\hline Stage II & $96(51.9)$ & 81.3 & $\ldots .$. & & & 83.93 & $\ldots .$. & & \\
\hline Stage III & $43(23.2)$ & 52.6 & 39.00 & & & & & & \\
\hline Stage I only & & & & .306 & 0.58 & \multirow{3}{*}{\multicolumn{2}{|c|}{$\begin{array}{l}\text { All censored, no } \\
\text { statistics shown }\end{array}$}} & 2.60 & 0.11 \\
\hline No chemo & $19(41.3)$ & 64.59 & & & & & & & \\
\hline Chemo & $27(58.7)$ & 95.29 & & & & & & & \\
\hline Stage II only & & & & 14.35 & $\leq 0.001$ & & & 0.152 & 0.697 \\
\hline No chemo & $20(20.8)$ & 50.76 & & & & 60.53 & & & \\
\hline Chemo & $76(79.2)$ & 86.91 & & & & 84.53 & & & \\
\hline Stage III only & & & & 3.87 & 0.049 & & & 0.313 & 0.576 \\
\hline No chemo & $2(4.7)$ & 17.50 & 17.00 & & & 12.00 & 6.00 & & \\
\hline Chemo & $41(95.3)$ & 54.36 & 68.00 & & & 50.33 & 46.00 & & \\
\hline
\end{tabular}

an important consideration in developing clinical trials. As well, standardization of staging investigations could alter this finding.

Triple negative breast cancer carries an inferior prognosis when compared to nonTNBC [10]. In this review we confirm previous findings that identify earlier stages of TNBC have better outcomes than later stages. Although this is intuitive, this finding is often lost in the lay literature. Hernandez et al. [11] identified an inferior prognosis in patients with lymph node positivity, but a 10 year overall survival of $84 \%$ in patients with T1, N0, stage I TNBC. This is similar to our findings. Almost one quarter of our TNBC patients presented with Stage 1 disease. It is important we are aware of this patient population, as their treatment recommendations are unclear and a source of a fair amount of controversy currently.

We were only able to identify a statistically significant difference in PFS for the receipt of chemotherapy in stage II patients. We suggest that the relative benefit of the receipt of chemotherapy in the stage I patient population would likely be smaller and therefore require a larger sample size. As well, as more personalized medicine develops in this field certain patient or tumor related factors may allow us to better select patient for this treatment. It is encouraging to note that the no relapses were seen after 2 years in the patients that received chemotherapy in the stage I patient population. The lack of statistical difference identified in the stage III population of patients likely is a result of the fact that $95 \%$ received chemotherapy, therefore too small a comparator arm.

It is interesting that radiotherapy also was found to be a predictor of outcome in the multi-variate analysis. There were no reported differences in the surgical management of the patients, but further investigation into this finding will be performed.

Because of lack of studies in the stage I TNBC, management of this stage of patients is still based on extrapolation from the higher stages of TNBC, and appropriately, there are concerns regarding over-treatment of this sub-population of early stage TNBC. A prospective trial examining this patient population would be helpful in answering this 
question. Certainly other factors such as BRCA status, tumor-infiltrating lymphocytes, and molecular profile will also be helpful in guiding treatment decisions. The adjusted Cox regression for progression-free and overall survival demonstrated that earlier stage, lower tumor grade and receipt of chemotherapy were the factors that predicted improved outcome in patients with TNBC.

This retrospective review supports current guidelines that recommend chemotherapy for all patients with triple negative breast cancer. It also emphasizes that patients with earlier stage breast cancer have a significantly better outcome than those with a more advanced stage. This is important in our discussion with patients with this diagnosis. The use of cisplatin and carboplatin to treat triple-negative breast cancers has demonstrated significant activity in triple negative breast cancer, and is currently being studied at our centre in the adjuvant setting, as well as other clinical trials in the United States. Initial findings suggest that neoadjuvant use of cisplatin results in high rates of complete pathological response in patients with triple-negative cancer [14]-[16].

\section{Conclusion}

In summary, the presenting stage of a patient with triple negative breast cancer is the most important predictor of progression free and overall survival. Patients with stage I do significantly better than those in the more advanced stages. Although this is intuitive to practicing physicians, this fact can be missed in patients presenting to the cancer centre, who often present with great fear based on their triple negative status. Although the prognosis of the triple negative phenotype is not as good as their non-TNBC counterparts, earlier presenting stage will do significantly better than later stages. It is important to be we are aware of this patient population, as their treatment recommendations are unclear and a source of a fair amount of controversy currently. Prospective studies focusing on stage I TNBC will be needed to answer this question. Standardized baseline staging for triple negative breast cancer patients will have to be developed. Ongoing research to identify certain patient subsets that are more likely to benefit from the platinums, as well as searching for targets for therapy in this patient subset is necessary.

\section{References}

[1] Perou, C.M. (2010) Molecular Stratification of Triple-Negative Breast Cancers. The Oncologist, 15, 39-48. http://dx.doi.org/10.1634/theoncologist.2010-s5-39

[2] Turner, N.C. and Reis-Filho, J.S. (2013) Tackling the Diversity of Triple-Negative Breast Cancer. Clinical Cancer Research: An Official Journal of the American Association for Cancer Research, 19, 6380-6288. http://dx.doi.org/10.1158/1078-0432.CCR-13-0915

[3] Tung, N.M. and Winer, E.P. (2015) Tumor-Infiltrating Lymphocytes and Response to Platinum in Triple-Negative Breast Cancer. Journal of Clinical Oncology: Official Journal of the American Society of Clinical Oncology, 33, 969-971. http://dx.doi.org/10.1200/JCO.2014.59.6031

[4] Dent, R., Trudeau, M., Pritchard, K.I., Hanna, W.M., Kahn, H.K., Sawka, C.A., et al. (2007) Triple-Negative Breast Cancer: Clinical Features and Patterns of Recurrence. Clinical Cancer Research: An Official Journal of the American Association for Cancer Research, 13, 
4429-4434. http://dx.doi.org/10.1158/1078-0432.CCR-06-3045

[5] Liedtke, C., Mazouni, C., Hess, K.R., Andre, F., Tordai, A., Mejia, J.A., et al. (2008) Response to Neoadjuvant Therapy and Long-Term Survival in Patients with Triple-Negative Breast Cancer. Journal of Clinical Oncology: Official Journal of the American Society of Clinical Oncology, 26, 1275-1281. http://dx.doi.org/10.1200/JCO.2007.14.4147

[6] Abdel-Wahab, O., Pardanani, A., Bernard, O.A., Finazzi, G., Crispino, J.D., Gisslinger, H., et al. (2012) Unraveling the Genetic Underpinnings of Myeloproliferative Neoplasms and Understanding Their Effect on Disease Course and Response to Therapy: Proceedings from the 6th International Post-ASH Symposium. American Journal of Haematology, 87, 562568. http://dx.doi.org/10.1002/ajh.23169

[7] Lin, N.U., Vanderplas, A., Hughes, M.E., Theriault, R.L., Edge, S.B., Wong, Y.N., et al. (2012) Clinicopathologic Features, Patterns of Recurrence, and Survival among Women with Triple-Negative Breast Cancer in the National Comprehensive Cancer Network. Cancer, 118, 5463-5472. http://dx.doi.org/10.1002/cncr.27581

[8] Lee, J.A., Kim, K.I., Bae, J.W., Jung, Y.H., An, H., Lee, E.S., et al. (2010) Triple negative Breast Cancer in Korea-Distinct Biology with Different Impact of Prognostic Factors on Survival. Breast Cancer Research and Treatment, 123, 177-187. http://dx.doi.org/10.1007/s10549-010-0998-5

[9] Iwase, H., Kurebayashi, J., Tsuda, H., Ohta, T., Kurosumi, M., Miyamoto, K., et al. (2010) Clinicopathological Analyses of Triple Negative Breast Cancer Using Surveillance Data from the Registration Committee of the Japanese Breast Cancer Society. Breast Cancer, 17, 118-124. http://dx.doi.org/10.1007/s12282-009-0113-0

[10] Hamm, C., El-Masri, M., Poliquin, G., Poliquin, V., Mathews, J., Kanjeekal, S., et al. (2011) A Single-Centre Chart Review Exploring the Adjusted Association between Breast Cancer Phenotype and Prognosis. Current Oncology, 18, 191-196. http://dx.doi.org/10.3747/co.v18i4.815

[11] Hernandez-Aya, L.F., Chavez-Macgregor, M., Lei, X., Meric-Bernstam, F., Buchholz, T.A., Hsu, L., et al. (2011) Nodal Status and Clinical Outcomes in a Large Cohort of Patients with Triple-Negative Breast Cancer. Journal of Clinical Oncology: Official Journal of the American Society of Clinical Oncology, 29, 2628-2634. http://dx.doi.org/10.1200/JCO.2010.32.1877

[12] Carey, L.A., Perou, C.M., Livasy, C.A., Dressler, L.G., Cowan, D., Conway, K., et al. (2006) Race, Breast Cancer Subtypes, and Survival in the Carolina Breast Cancer Study. JAMA, 295, 2492-502. http://dx.doi.org/10.1001/jama.295.21.2492

[13] Bauer, K.R., Brown, M., Cress, R.D., Parise, C.A. and Caggiano, V. (2007) Descriptive Analysis of Estrogen Receptor (ER)-Negative, Progesterone Receptor (PR)-Negative, and HER2-Negative Invasive Breast Cancer, the So-Called Triple-Negative Phenotype: A Population-Based Study From the California Cancer Registry. Cancer, 109, 1721-1728. http://dx.doi.org/10.1002/cncr.22618

[14] Byrski, T., Huzarski, T., Dent, R., Gronwald, J., Zuziak, D., Cybulski, C., et al. (2009) Response to Neoadjuvant Therapy with Cisplatin in BRCA1-Positive Breast Cancer Patients. Breast Cancer Research and Treatment, 115, 359-363. http://dx.doi.org/10.1007/s10549-008-0128-9

[15] Sikov, W.M., Berry, D.A., Perou, C.M., Singh, B., Cirrincione, C.T., Tolaney, S.M., et al. (2015) Impact of the Addition of Carboplatin and/or Bevacizumab to Neoadjuvant OncePer-Week Paclitaxel Followed by Dose-Dense Doxorubicin and Cyclophosphamide on Pathologic Complete Response Rates in Stage II to III Triple-Negative Breast Cancer: CALGB 40603 (Alliance). Journal of Clinical Oncology: Official Journal of the American Society of Clinical Oncology, 33, 13-21. http://dx.doi.org/10.1200/JCO.2014.57.0572 
[16] von Minckwitz, G., Schneeweiss, A., Loibl, S., Salat, C., Denkert, C., Rezai, M., et al. (2014) Neoadjuvant Carboplatin in Patients with Triple-Negative and HER2-Positive Early Breast Cancer (GeparSixto; GBG 66): A Randomised Phase 2 Trial. The Lancet Oncology, 15, 747-756. http://dx.doi.org/10.1016/S1470-2045(14)70160-3

\section{List of Abbreviations}

TNBC: Triple Negative Breast Cancer

Non-TNBC: Non-Triple Negative Breast Cancer

PET: Positron Emission Tomography

ER: Estrogen Receptor

PR: Progesterone Receptor

HER 2: Human Epidermal Growth Factor Receptor

dose dense ACT: Dose dense Adriamycin, Cyclosphosphamide, Taxol

5FU: 5 Fluoro-Uracil

FEC-D: 5 Fluoro-Uracil, Epirubicin, Cyclosphosphamide-Docetaxel

HR: Hazard Ratio

Submit or recommend next manuscript to SCIRP and we will provide best service for you:

Accepting pre-submission inquiries through Email, Facebook, LinkedIn, Twitter, etc.

A wide selection of journals (inclusive of 9 subjects, more than 200 journals)

Providing 24-hour high-quality service

User-friendly online submission system

Fair and swift peer-review system

Efficient typesetting and proofreading procedure

Display of the result of downloads and visits, as well as the number of cited articles

Maximum dissemination of your research work

Submit your manuscript at: http://papersubmission.scirp.org/

Orcontact jct@scirp.org 